\title{
STRESS CONCENTRATION AND DISTRIBUTION AT TRIPLE JUNCTION PORES OF THREE-FOLD SYMMETRY IN CERAMICS
}

\author{
A.B. Vakaeva'1, S.A. Krasnitckii ${ }^{2,3}$, A.M. Smirnov³ ${ }^{3}$ M.A. Grekov' ${ }^{1}$ \\ and M.Yu. Gutkin ${ }^{2,3,4}$ \\ ${ }^{1}$ St. Petersburg State University, Universitetskaya nab.7/9, St. Petersburg, 199034, Russia \\ ${ }^{2}$ Institute of Problems of Mechanical Engineering, Russian Academy of Sciences, Bolshoi 61, Vasil. Ostrov, \\ St. Petersburg, 199178, Russia \\ ${ }^{3}$ ITMO University, Kronverkskii pr. 49, St. Petersburg, 197101, Russia \\ ${ }^{4}$ Peter the Great St. Petersburg Polytechnic University, Polytekhnicheskaya 29, St. Petersburg, 195251 Russia \\ Received: May 12, 2018

\begin{abstract}
The stress concentration and distribution around a triple-junction pore of three-fold symmetry in a polycrystalline ceramic material is considered. The perturbation method in the theory of plane elasticity is used to solve the problem of a nearly circular pore of three-fold symmetry under remote loading in the first approximation. The solution was specified to the uniaxial tension of convex and concave rounded triangular pores. The stress concentration on the pore surface and the stress distribution in vicinity of the pore along its symmetry axes are studied and discussed in detail. The numerical results, issued from the first-order approximation analytical solution, are compared with those of finite-element calculations.
\end{abstract}

\section{INTRODUCTION}

Most of ceramics and ceramic composites contain pores which are considered as one of the main features in the structure of ceramic materials [1-4]. The type, size and distribution of pores in ceramics strongly depend on the technology used for their synthesis and further processing. The common place is the presence of pores at grain boundaries (GBs) and their triple junctions (TJs). In these cases, pores make a great contribution to the brittleness of ceramics because they play the role of stress concentrators and sources for intergranular cracking. On the other hand, in nanoceramics with nanoscopic pores [4,5], the latter can play the role of the sources for GB gliding through stimulation of local shear events or emission of gliding GB dislocations [612]. Anyway, the study of stress concentration on pores located at GBs and TJs is of primary impor- tance for developing theoretical models of stress relaxation, plastic deformation and fracture in ceramics under external loading.

In the context of stress concentration on pores, their actual shape is obviously relevant. Many authors considered various shapes of pores and holes, from classical two-dimensional (2D) holes in thin plates (see, for example, books $[13,14]$ and references therein) to more recent studies of nearly circular and irregular 2D holes [15-24]. The corresponding elastic problems were solved by either the conformal mapping (analytical $[13-15,17]$ and numerical $[19,20]$ ) or perturbation methods $[14,16,21$ 23] with special attention to the stress concentration on the hole surface $[13,14,16,18,22,24]$, compressibility of holes under hydrostatic compression $[15,21]$ and the hole-shape effect on the effective properties of porous materials [17,19-21, 23,25].

Corresponding author: M.Yu. Gutkin, e-mail: m.y.gutkin@gmail.com 


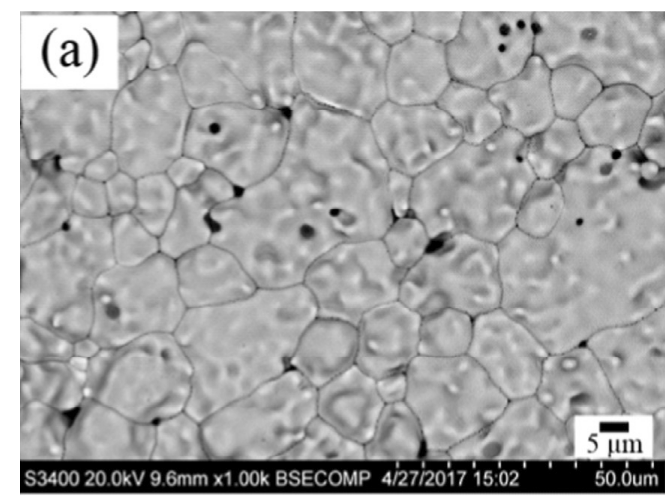

(b)

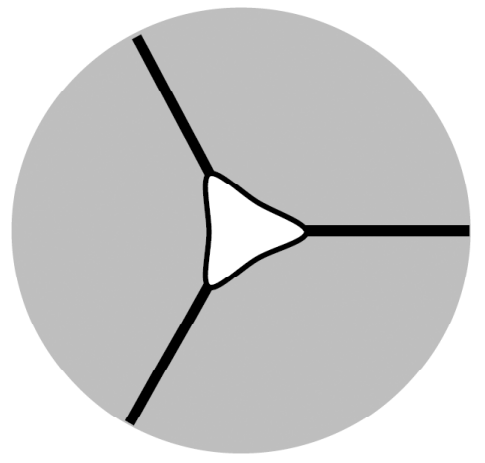

Fig. 1. (a) An example of intra- and intergranular porosity in $12 \mathrm{CaO}-88 \mathrm{ZrO}_{2}(12 \mathrm{CSZ}$, mol.\%) ceramics [28]; @ Elsevier, with permission. Most of the intergranular pores are of nearly triangular shape and are located at triple junctions of grain boundaries. (b) Sketch of an idealized triple-fold symmetry pore in such a triple junction.

In particular, the perturbation method, developed in Refs. [22,24,26,27], occurred rather effective in describing stress concentration on the boundaries of holes and inhomogeneities of nearly circular shape in any order of approximation within both the classical [22] and surface/interface $[24,26,27]$ theory of elasticity.

In the present work, we apply the perturbation method [22] to the case of nearly circular pores of three-fold symmetry, which often occur at TJs in ceramic materials [1-5]. One example of typical ceramic microstructure is shown in Fig. 1a where a SEM image of $12 \mathrm{CaO}-88 \mathrm{ZrO}_{2}$ (12CSZ, mol.\%) ceramics [28] clearly demonstrates both intra- and intergranular porosity with $\mathrm{TJ}$ pores of nearly triangular shape (see also the sketch in Fig. 1b). Similar pores were studied by Givoli and Elishakoff [16] with using the perturbation method with terms up to the second order of approximation for the case of uniform radial tension at infinity and by Ekneligoda and Zimmerman [21] who used the same method with terms up to the forth order of approximation for the case of hydrostatic stress at infinity. In our turn, we take the first-order approximation solution for the case when the pore is under remote normal and shear stresses. In doing so, we specially concentrate on the case of uniaxial tension and calculate the stress concentration on the pore surface and the stress distribution in vicinity of the pore along its symmetry axes. We also compare our results with those obtained by finite-element calculations within the Academic Research version of the ANSYS program.

\section{STATEMENT OF THE PROBLEM}

Consider a pore placed at the equilibrium $\mathrm{TJ}$, in which three GBs meet under the angles $120^{\circ}$ in a macroscopic sample of ceramics under remote homogeneous loading. We approximate the pore shape with the following parametric equation in polar coordinates $(r, \theta)$

$\rho=1+\varepsilon \cos 3 \theta$,

where $\rho$ is the normalized position vector (in units of $R$ ) describing the pore boundary, $\theta$ is the polar angle, $R$ is the radius of the circle roughly corresponding to the pore shape, $\varepsilon$ is a small parameter which defines the maximum deviation of the pore boundary from the circular one, $\varepsilon>0$ and $\varepsilon<<1$. At $\varepsilon \rightarrow 0$ Eq. (1a) gives the unit circle. The geometry of pore is shown in Fig. 2 for two different values of $\varepsilon$, $\varepsilon=0.1$ and 0.2 . As is seen, the pore has three axes of symmetry, which are assumed to lie in the GB planes (Fig. 1b).

Within the classical theory of elasticity [13], we consider an infinite elastic body $\Omega$ with a pore which is bounded by the free surface $\Gamma$ determined by Eq. (1a). We treat the stress state around the pore as plain strain and introduce a dimensionless complex variable $z=x_{1}+i x_{2}$, where $i=\sqrt{-1}$. In complex notation, the pore boundary is given by

$$
\zeta=\left.z\right|_{r=\rho}=\rho \exp (i \theta)=(1+\varepsilon \cos 3 \theta) s, \quad \zeta \in \Gamma,
$$

where $s=\exp (i \theta)$.

The boundary conditions on the pore free surface $(\zeta \in \Gamma)$ could be represented as complex combination

$\sigma_{n}(\zeta)=\left[\sigma_{n n}+i \sigma_{n t}\right]_{r=\rho}=0$,

where $\sigma_{n}$ is the traction at the area with the normal $\mathbf{n}, \sigma_{n n}$ and $\sigma_{n t}$ are the normal and shear components, respectively, of the stress tensor with respect to the pore boundary.

Let the pore is under remote loading. At infinity, the stresses $\sigma_{i j}^{\infty}(i, j=1,2)$ are determined by 
(a)

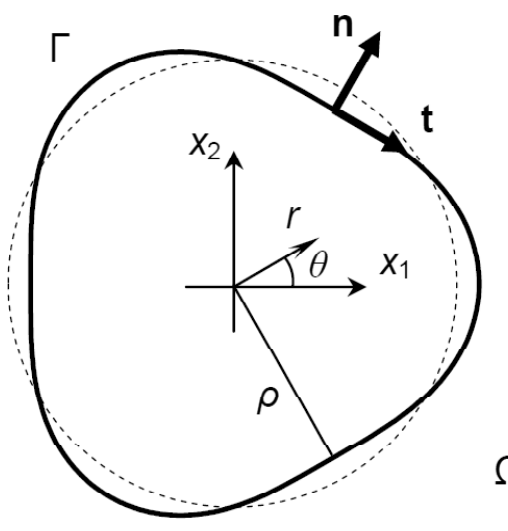

(b)

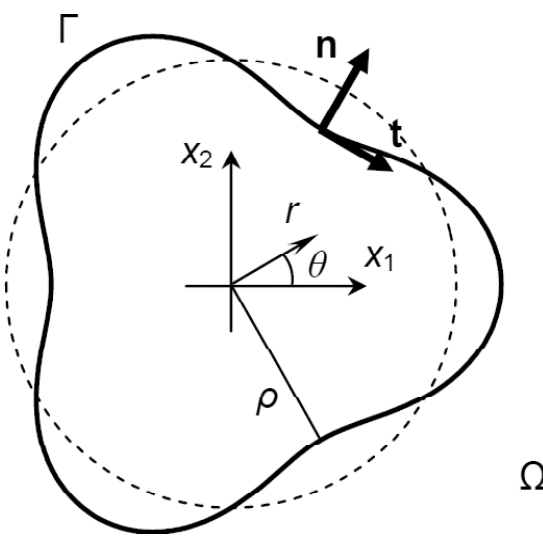

Fig. 2. Pore boundary for different values of deviation $\varepsilon$ from the unit circle: (a) $\varepsilon=0.1$ and (b) $\varepsilon=0.2$. Here $\mathbf{n}$ and $\mathbf{t}$ are the unit normal and tangential vectors, respectively, to the pore boundary. The Cartesian $\left(x_{1}, x_{2}\right)$ and polar $(r, \theta)$ normalized coordinates in units $R$ are shown.

$\lim _{z \rightarrow \infty} \sigma_{i j}=\sigma_{i j}^{\infty}=s_{i j}$.

It is also suggested that rotation vanishes at infinity, in which case the rotation angle $\omega$ satisfies the condition

$\lim _{z \rightarrow \infty} \omega=0$.

At an arbitrary point of the $z$-plane, the stress components can be expressed through the Goursat-Kolosov complex potentials $\Phi$ and $\Upsilon[13,22]$, which are holomorphic, respectively, in the domain $\Omega$ outside of the pore boundary and in the finite region $D=\left\{z: \bar{z}^{-1} \in \Omega\right\}$ with the boundary $\Gamma_{1}$ which is symmetrical to the boundary $\Gamma$ with respect to the unit circle:

$\sigma_{n n}+\sigma_{t t}=\sigma_{r r}+\sigma_{\theta \theta}=2[\Phi(z)+\overline{\Phi(z)}]=4 \operatorname{Re}[\Phi(z)]$,

$\left(\sigma_{t t}-\sigma_{n n}-2 i \sigma_{n t}\right) e^{2 i \alpha}=\sigma_{\theta \theta}-\sigma_{r r}-2 i \sigma_{r \theta}=-2\left[\frac{1}{\bar{z}^{2}}\left(\overline{\Phi(z)}+\Upsilon\left(\frac{1}{\bar{z}}\right)\right)+\left(z-\frac{1}{\bar{z}}\right) \overline{\Phi^{\prime}(z)}\right] e^{-2 i \theta}$,

where $\alpha$ is the angle between the normal $\mathbf{n}$ of the area and the radius vector of the point $z$.

Subtracting Eq. (4a) from Eq. (4b) and passing to the limit for $z \rightarrow \zeta$ with taking Eqs. (3) into account, we get the following boundary equation for definition of the complex potentials $\Phi$ and $\Upsilon$ :

$\sigma_{n}(\zeta)=\Phi(\zeta)+\overline{\Phi(\zeta)}+\frac{\rho^{\prime}-i \rho}{\rho^{\prime}+i \rho}\left[\frac{1}{\bar{\zeta}^{2}}\left(\overline{\Phi(\zeta)}+\Upsilon\left(\frac{1}{\bar{\zeta}}\right)\right)+\left(\zeta-\frac{1}{\bar{\zeta}}\right) \overline{\Phi^{\prime}(\zeta)}\right] \bar{s}^{2}$,

where $\Phi(\zeta)=\lim _{z \rightarrow \zeta} \Phi(z)$ when $z \in \Omega$ and $\Upsilon(\zeta)=\lim _{z \rightarrow \zeta} \Upsilon(z)$ when $z \in \mathrm{D}$.

\section{FIRST-ORDER APPROXIMATION SOLUTION}

Following the boundary perturbation procedure [22,26,27], the complex potentials $\Phi(z), \Upsilon(z)$ and the solution of the problem as a whole are sought in terms of power series in a small parameter $\varepsilon$ :

$\Phi(z)=\sum_{n=0}^{+\infty} \frac{\varepsilon^{n}}{n !} \Phi_{n}(z), \Upsilon(z)=\sum_{n=0}^{+\infty} \frac{\varepsilon^{n}}{n !} \Upsilon_{n}(z)$.

In turn, we expand the limit values of the functions $\Phi_{n}(z)$ on $\Gamma$ and $\Upsilon_{n}(z)$ on $\Gamma_{1}$, and all other functions in boundary equation (5) in the corresponding Taylor series near the unit circle $z=s$ in the variable $s$ considered as a parameter, 


$$
\begin{aligned}
& \Phi(\zeta)=\sum_{n=0}^{+\infty} \frac{\varepsilon^{n}}{n !} \sum_{k=0}^{+\infty} \frac{(\varepsilon f(s) s)^{k}}{k !} \frac{d^{k}}{d s^{k}} \Phi_{n}(s), \Upsilon\left(\frac{1}{\zeta}\right)=\sum_{n=0}^{+\infty} \frac{\varepsilon^{n}}{n !} \sum_{k=0}^{+\infty} \frac{(\varepsilon f(\bar{s}) \bar{s})^{k}}{k !} \frac{d^{k}}{d \bar{s}^{k}} \Upsilon_{n}\left(\frac{1}{\bar{s}}\right) . \\
& \frac{\rho^{\prime}-i \rho}{\rho^{\prime}+i \rho}=-1-2 i \delta^{\prime} \sum_{k=0}^{+\infty}\left(i \delta^{\prime}-\delta\right)^{k}, \zeta-\frac{1}{\bar{\zeta}}=s\left[\delta-\sum_{k=1}^{+\infty}(-\delta)^{k}\right], \frac{1}{\bar{\zeta}^{2}}=s^{2} \sum_{k=0}^{+\infty}(k+1)(-\delta)^{k},
\end{aligned}
$$

where $\delta=\varepsilon f(s)$ and $f(s)=\left(s^{3}+s^{-3}\right) / 2=\cos 3 \theta$.

Substituting Eqs. (6-8) into Eq. (5), we obtain a sum of coefficients at the same power terms $\varepsilon^{n}(n=0,1, \ldots)$, which is equal to zero. Then we come to the Riemann-Gilbert boundary value problems on the jump of holomorphic functions $\Xi_{n}(z)$ for the $n$-order approximation

$\Xi_{n}^{+}(s)-\Xi_{n}^{-}(s)=q_{n}(s),|s|=1$,

where $\Xi_{n}^{ \pm}(s)=\lim _{\mid z \rightarrow \rightarrow \neq 0} \Xi_{n}(z)$ and $q_{n}$ are known functions which depend on all previous approximations and the conditions on infinity (3). The piecewise holomorphic functions $\Xi_{n}(z)$ are defined as

$\Xi_{n}(z)=\left\{\begin{array}{l}\Phi_{n}(z),|z|>1 \\ \Upsilon_{n}(z),|z|<1\end{array}\right.$

According to [13], the solution of the problem can be written in terms of Cauchy type integrals

$$
\Xi_{n}(z)=\frac{1}{2 \pi i} \int_{\left|\zeta_{0}\right|=1} \frac{q_{n}(\zeta)}{\zeta-z} \mathrm{~d} \zeta+R_{n}(z)
$$

where $R_{0}=D_{1}+D_{2} z^{2}, R_{n}=0, n=1,2 \ldots$.

The zero-order and first-order approximations of the function $q_{n}$ are given by formulas

$$
q_{0}=0 \text {, }
$$

$q_{1}=-\bar{s} f(\bar{s}) \frac{\mathrm{d}}{\mathrm{d} \bar{s}} \Upsilon_{0}\left(\frac{1}{\bar{s}}\right)+s f(s) \frac{\mathrm{d}}{\mathrm{d} s} \Phi_{0}(s)+2\left(f(s)-i f_{\theta}^{\prime}(s)\right)\left(\overline{\Phi_{0}(s)}+\Upsilon_{0}\left(\frac{1}{\bar{s}}\right)\right)-2 \bar{s} f(s) \overline{\Phi_{0}^{\prime}(s)}$.

Following the algorithm constructed by Bashkankova et al. [22], the complex potentials in the zeroorder approximation, which correspond to the solution of the appropriate boundary value problem for the circular pore, are determined as

$\Phi_{0}(z)=\Upsilon_{0}(z)=D_{1}+\bar{D}_{2} z^{-2}$.

The complex potentials in the first-order approximation for the nearly circular pore of the shape determined by Eq. (1) read

$$
\begin{aligned}
& \Phi_{1}(z)=\frac{4}{z^{3}}\left[D_{1}+\frac{\bar{D}_{2}}{z^{2}}\right], \\
& \Upsilon_{1}(z)=2 \bar{D}_{2} z+6 D_{2} z^{5}+8 D_{1} z^{3} .
\end{aligned}
$$

The unknown constants $D_{1}$ and $D_{2}$ are defined from the conditions at infinity (3) as follows

$$
\begin{aligned}
& D_{1}=\frac{S_{11}+S_{22}}{4}, \\
& D_{2}=\frac{S_{22}-s_{11}+2 i s_{12}}{2} .
\end{aligned}
$$




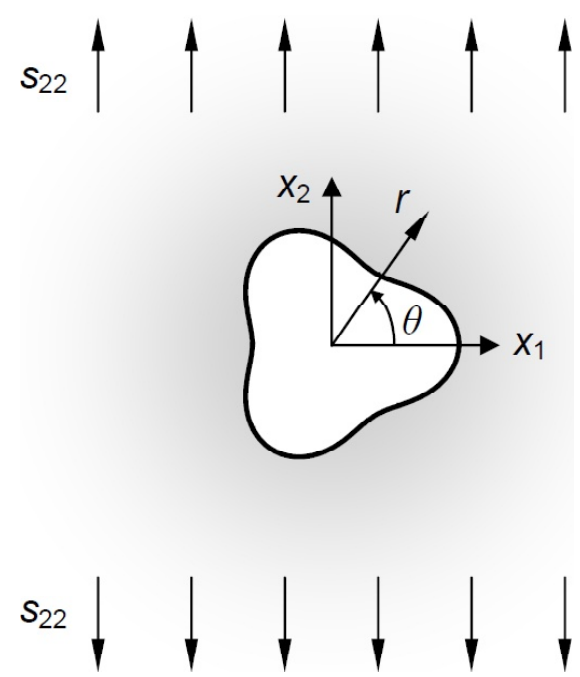

Fig. 3. Loading scheme for an elastic medium containing a cylindrical pore of three-fold symmetry under uniaxial tension $s_{22}$.

Thus, the stress state around the nearly circular pore under consideration is finally defined by formulas (4a,b) with (14) and (15).

\section{RESULTS AND DISCUSSION}

In the context of our model of a TJ pore in a ceramic material, it is of interest to analyze in detail the case of uniaxial tension. This will allow us to apply in the future the obtained solution to theoretical models of crack nucleation in the GB plane with $\theta=0$ and $G B$ dislocation emission in the other GB plane with $\theta=2 \pi / 3$. Thus, let us consider the case of the $x_{2}$ axial tension (Fig. 3). In doing so, we suppose that $s_{11}$ and $s_{12}$ are equal to zero, while $s_{22}$ is a positive constant.

Fig. 4 shows the distribution of the tangential stress $\sigma_{\text {tt }}$ over the free surface of the pore for three different shapes of its boundary, which are characterized by $\varepsilon=0$ (the circular pore), 0.1 (the convex rounded triangular pore) and 0.2 (the concave rounded triangular pore). As is seen from Fig. 4 , the stress concentration at the point $\theta=0$, which is crucial for crack generation along the corresponding $\mathrm{GB}$, increases with $\varepsilon$ and reaches the values $\sim 4.5$ and $\sim 6.0$ for $\varepsilon=0.1$ and 0.2 , respectively, that is obviously explained by the corresponding increase in the pore curvature at this point. On the opposite side of the pore, at the point $\theta=\pi$, the stress concentration decreases with $\varepsilon$ and reaches the values $\sim 1.5$ and $\sim 0.0$ for $\varepsilon=0.1$ and 0.2 , respectively, in contrast with classical value of 3 for $\theta=\pi$. Interestingly, at the points $\theta \approx 23^{\circ}, 58^{\circ}, 90^{\circ}, 118^{\circ}$, and $158^{\circ}$ (for the 'top' half of the pore), the stress value remains roughly constant and does not depend on the value of $\varepsilon$ (Fig. 4). Rather high stress concentration ( 3.55) is also observed for $\varepsilon=0.2$ at the point $\theta=13 \pi / 16 \approx 146^{\circ}$, where the tangent to the pore boundary coincides with the axis of tension.

We have verified our numerical results taken from the above first-approximation analytical solution by comparing with those of finite element calculations within the ANSYS software. The finite element model

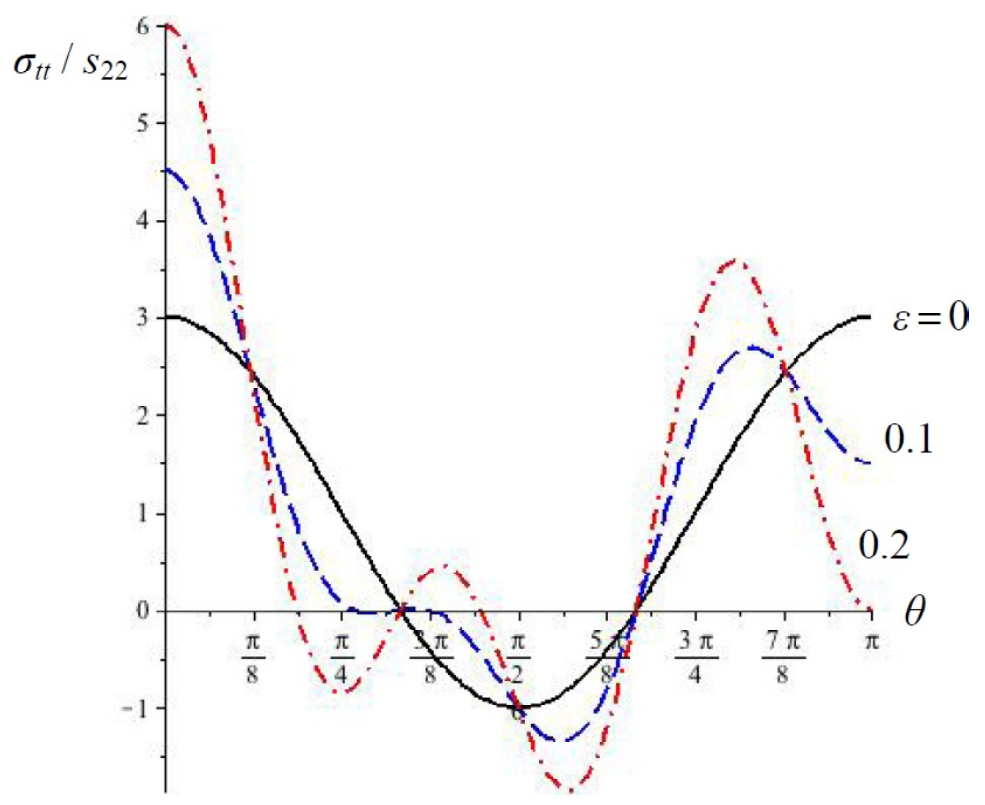

Fig. 4. Distribution of the tangential stress $\sigma_{t t}(r=\rho, \theta)$ over the 'top' half of the pore boundary for different values of the parameter $\varepsilon$ : $\varepsilon=0$ (the solid curve), 0.1 (the dashed curve), and 0.2 (the dashed-and-dotted curve). 
(a)

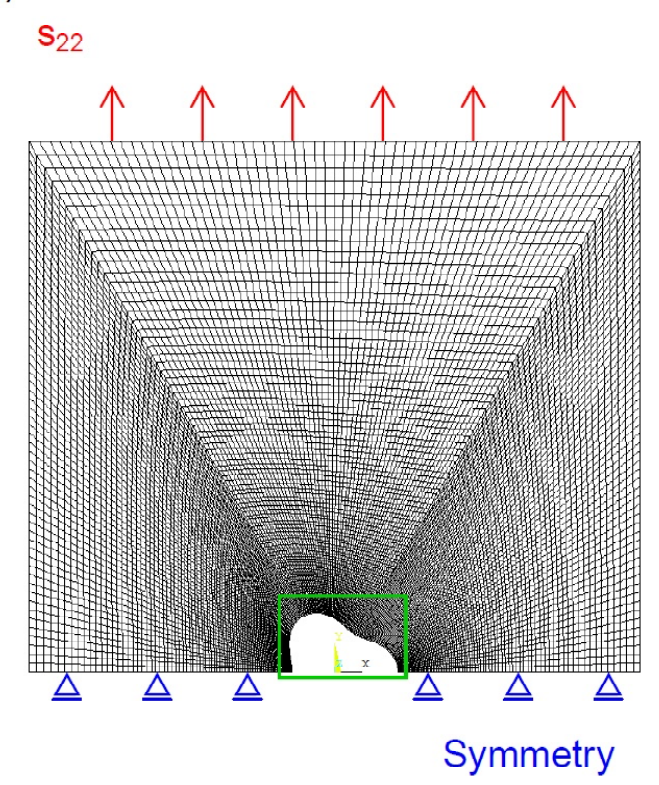

(b)

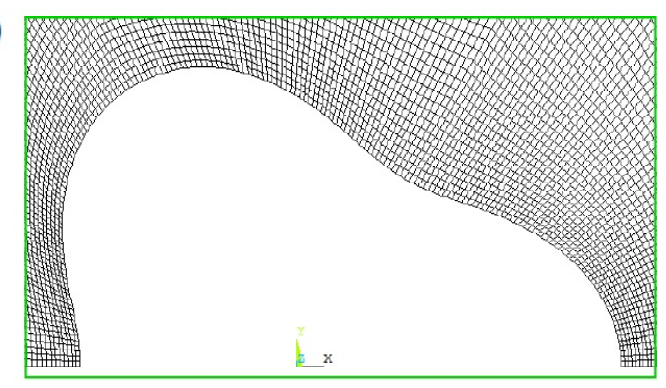

(c)

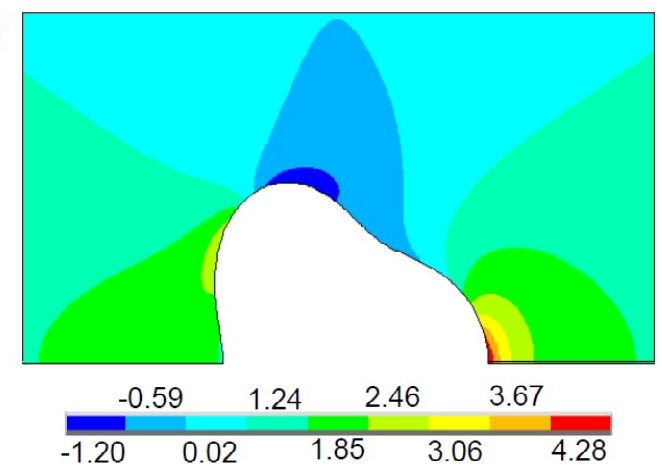

Fig. 5. (a) Finite element model of an elastic body with a concave rounded triangular pore $(\varepsilon=0.2)$; the symmetry of the problem is taken into account. (b) A fragment of the finite element model near the pore. (c) Distribution of the stress component $\sigma_{\theta \theta} / s_{22}$ around the pore.

of an elastic body with concave rounded triangular pore is shown in Figs. $5 \mathrm{a}$ and $5 \mathrm{~b}$. The model is built of parabolic elements which allow us to approximate the pore boundary with high accuracy. The symmetry of pore geometry and loading is taken into account through prohibiting the normal displacement on the bottom surface of the model. The size of the model is large enough compared to the pore size, i.e. the stresses $\sigma_{11}$ and $\sigma_{12}$ vanish far from the pore, while $\sigma_{22} \rightarrow s_{22}$ there. Fig. 5c shows a map of stress distribution around the pore, which illustrates the stress concentration phenomena by means of the finite element method.

Bearing in mind possible subsequent application of the above analytical solution to theoretical modeling of crack generation at and GB dislocation emission from the TJ pore along the adjacent GBs, it is of interest to consider the stress distribution along the lines $(r>\rho, \theta=0)$ and $(r>\rho, \theta=2 \pi / 3)$ which are the symmetry axes of the pores. Fig. 6 shows the plots $\sigma_{\theta \theta}(r), \sigma_{r r}(r)$ and $\sigma_{r \theta}(r)$ built numerically with the help of both the first-approximation analytical solution (solid curves) and finite element calculations (dots) for convex ( $\varepsilon=0.1)$ and concave $(\varepsilon=0.2)$ rounded triangular pores. As is seen, the first-approximation analytical solution gives the results which are qualitatively and often quantitatively similar to those of finite element calculations. Some disagreement in values is explained by the necessity to include into consideration the next orders of approximation. It is natural that this disagreement is noticeably smaller for the smaller value of $\varepsilon$. In fact, the fist-order approximation gives overestimated results. The largest discrepancy between the first-order approximation and the finite element results is observed at the pore boundary. However, the discrepancy sharply decreases with the distance from the pore. Nevertheless the next orders of approximation are desired for theoretical modeling of crack and dislocation generation.

The general conclusion from Fig. 6 is that the stress concentration effect due to the pores under consideration increases with $\varepsilon$ value and is significant in the region of approximately $r<3$. Outside this region, this effect becomes negligibly small.

\section{CONCLUSIONS}

In summary, we considered stress concentration and distribution around a triple-junction pore of threefold symmetry in a polycrystalline ceramic material. In doing so, we used the perturbation method in the theory of plane elasticity to solve the problem of a nearly circular pore of three-fold symmetry under remote loading in the first approximation. The solution was specified to the uniaxial tension of convex and concave rounded triangular pores which were characterized by small parameter $\varepsilon$ defining 

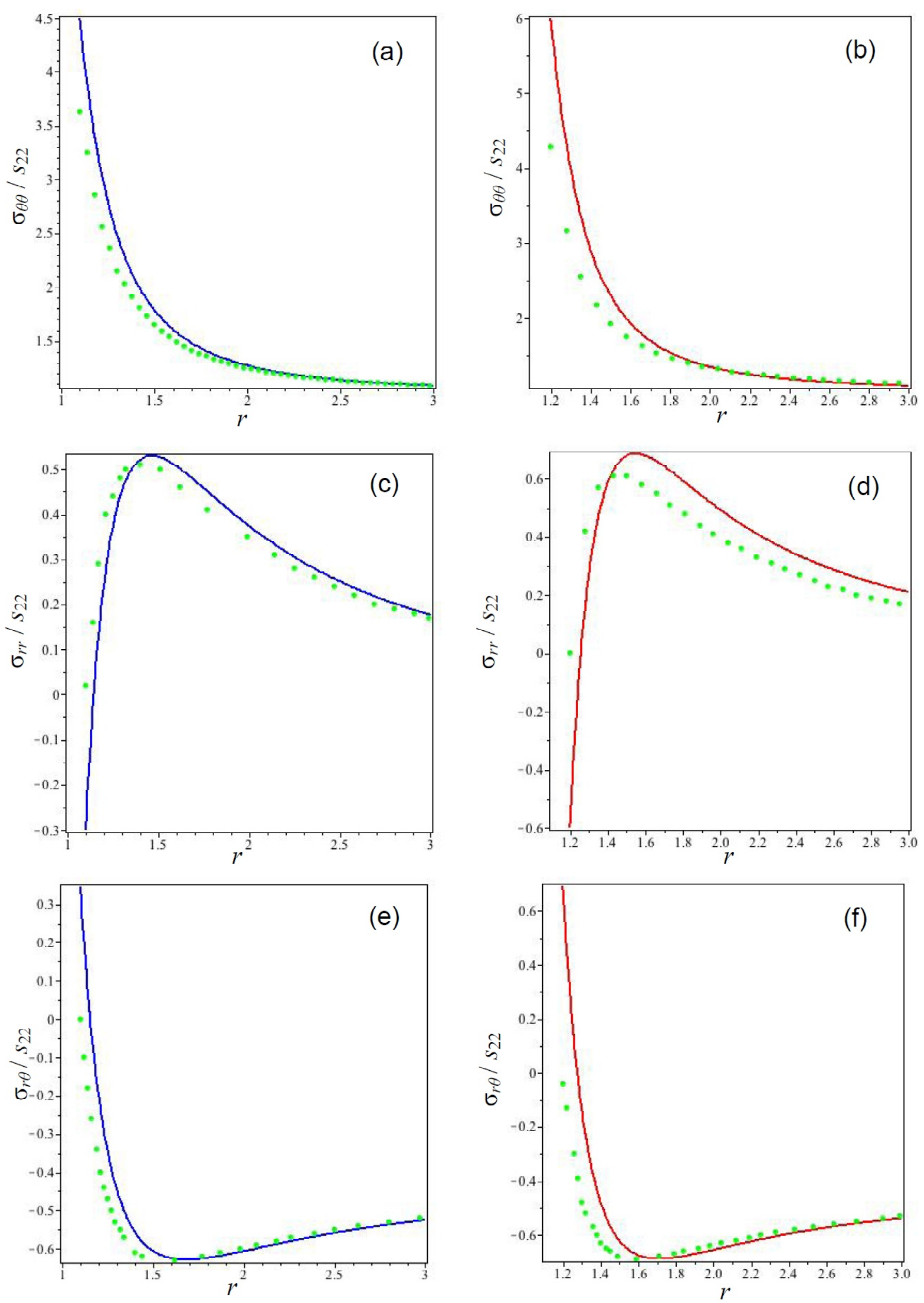

Fig. 6. Stress distribution along the lines $(a-d)(r>\rho, \theta=0)$ and $(e, f)(r>\rho, \theta=2 \pi / 3)$ for different values of $\varepsilon$ : $(\mathrm{a}, \mathrm{c}, \mathrm{e}) \varepsilon=0.1$ and $(\mathrm{b}, \mathrm{d}, \mathrm{f}) \varepsilon=0.2$. The stress components $(\mathrm{a}, \mathrm{b}) \sigma_{\theta \theta}(r, \theta=0),(\mathrm{c}, \mathrm{d}) \sigma_{r r}(r, \theta=0)$, and $(\mathrm{e}, \mathrm{f}) \sigma_{r \theta}(r, \theta=$ $2 \pi / 3$ ) were calculated numerically from the first-order approximation analytical solution (solid curves) and the finite element solution (dots). 
the maximum deviation of the pore boundary from the circular one with the $\varepsilon$ values 0.1 and 0.2 , respectively. The stress concentration on the pore surface and the stress distribution in vicinity of the pore along its symmetry axes were studied and discussed in detail. The numerical results, issued from the first-approximation analytical solution, were compared with the results of finite-element calculations within the ANSYS software.

It is shown that the maximum value of the tangential stress concentration increases with $\varepsilon$ and reaches the values $\sim 4.5$ and $\sim 6.0$ for $\varepsilon=0.1$ and 0.2 , respectively, in contrast with classical value of 3 for $\varepsilon=0$. Rather high stress concentration $(\sim 3.55)$ is also observed for $\varepsilon=0.2$ at the other point, where the tangent to the pore boundary coincides with the axis of tension. Our consideration of stress distribution along the symmetry axes of the pore has shown that the stress concentration effect due to the pores under consideration increases with $\varepsilon$ value and is significant in the region of approximately $r<3$. Outside this region, this effect becomes negligibly small.

In general, our finite-element calculations qualitatively coincide with the aforementioned conclusions although the quantitative agreement is not always good enough. Some disagreement in values, which is noticeably smaller for the smaller value of $\varepsilon$, requires to include next orders of approximation in the analytical description of the model. The accuracy of the fist-order approximation seems to be not enough for physical applications, so the next orders of approximation are desired for theoretical modeling of intergranular cracking and plasticity in close vicinity of TJ pores in ceramics.

\section{ACKNOWLEDGEMENTS}

A.B. Vakaeva and M.A. Grekov acknowledge the Russian Foundation for Basic Research (RFBR, project 18-01-00468, Mechanics of surface phenomena, superficial and subsurface defects in a solid body) for the support in providing the analytical calculations of stress distribution around a triple-junction pore.

S.A. Krasnitckii, A.M. Smirnov and M.Yu. Gutkin acknowledge the Russian Science Foundation (RSF, project 18-19-00255, Mechanics of bulk functional nanoceramics and ceramic nanocomposites) for the support in the invention of the theoretical model of triple junction pores in ceramics and numerical finite-element modeling of the corresponding problem.

\section{REFERENCES}

[1] C.B. Carter and M.G. Norton, Ceramic Materials: Science and Engineering, $2^{\text {nd }}$ Edition (Springer, New York, 2013).

[2] M.H. Rahaman, Ceramic Processing, $2^{\text {nd }}$ Edition (CRC Press, Taylor \& Francis Group, Boca Raton - London - New York, 2017).

[3] W.D. Kaplan and A. Avishai, In: Ceramic matrix composites; Microstructure, properties and applications, ed. by I.M. Low (Woodhead Publishing Limited, Cambridge, England, 2006), p. 285.

[4] A.V. Ragulya // Advances in Applied Ceramics 107 (3) (2008) 118.

[5] Y. Ishida, H. Ichinose, T. Kizuka and K. Suenaga // NanoStruct. Maters. 6 (1995) 115.

[6] A.P. Sutton and R.W. Balluffi, Grain Boundaries in Crystalline Materials (Oxford Sci., Oxford, 1996).

[7] H. Conrad and J. Narayan // Scr. Mater. 42 (2000) 1025.

[8] K.A. Padmanabhan and H. Gleiter // Mater. Sci. Eng. A 381 (2004) 28.

[9] A.A. Fedorov, M.Yu. Gutkin and I.A. Ovid'ko // Acta Mater. 51 (2003) 887.

[10] M.Yu. Gutkin, I.A. Ovid'ko and N.V. Skiba // Acta Mater. 52 (2004) 1711.

[11] S.V. Bobylev, M.Yu. Gutkin and I.A. Ovid'ko // Phys. Rev. B73 (2006) 064102.

[12] T. He, J. Zhou and H. Liu // J. Nanopart. Res. 17 (2015) 380.

[13] N.I. Muskhelishvili, Some Basic Problems of the Mathematical Theory of Elasticity (Groningen, Noordhoff, 1963).

[14] G.N. Savin, Stress Distribution Around Holes (NASA TT F-607, Washington, 1970).

[15] R.W. Zimmerman // J. Appl. Mech. 53 (1986) 500.

[16] D. Givoli and I. Elishakoff // J. Appl. Mech. 59 (2S) (1992) S65.

[17] M. Kachanov, I. Tsukrov and B. Shafiro // Appl. Mech. Rev. 47 (1) (1994) S151.

[18] K.R.Y. Simha and S.S. Mohapatra // Sādhanā 23 (4) (1998) 393.

[19] I. Tsukrov and J. Novak // Int. J. Sol. Str. 39 (2002) 1539.

[20] I. Tsukrov and J. Novak // Int. J. Sol. Str. 41 (2004) 6905.

[21] T.C. Ekneligoda and R.W. Zimmerman // J. Appl. Mech. 75 (2008) 011015. 
[22] E.A. Bashkankova, A.B. Vakaeva and M.A. Grekov // Mechanics of Solids 50(2) (2015) 198.

[23] G. Bruno and M. Kachanov // J. Am. Ceram. Soc. 99 (12) (2016) 3829.

[24] M.A. Grekov, S.A. Kostyrko and A.B. Vakaeva // AIP Conf. Proc. 1909 (2017) 020062.

[25] M. Kachanov and I. Sevostianov, Micromechanics of Materials, with Applications (Springer, 2018).

[26] M.A. Grekov and A.B. Vakaeva, In: Proceedings of the 7 th International
Conference on Coupled Problems in Science and Engineering (COUPLED PROBLEMS 2017), ed. by M. Papadrakakis, E. Oñate and B. Schrefler (Intenational Center for Numerical Methods in Engineering, CIMNE, 2017), p. 963.

[27] A.B. Vakaeva and M.A. Grekov // AIP Conf. Proc. 1959 (2018) 070036.

[28] O.Yu. Kurapova, O.V. Glumov, I.V. Lomakin, S.N. Golubev, M.M. Pivovarov, J.V.

Krivolapova and V.G. Konakov // Ceramics International 44 (2018) 15464. 